\title{
Dynamics of blood electrolytes in repeated hyper- and/or hypoglycaemic events in patients with type 1 diabetes
}

\author{
A. Caduff • H. U. Lutz • L. Heinemann • \\ G. Di Benedetto $\cdot$ M. S. Talary $\cdot$ S. Theander
}

Received: 1 December 2010 /Accepted: 9 May 2011 / Published online: 15 June 2011

(C) Springer-Verlag 2011

\begin{abstract}
Aims/hypothesis Electrolyte disturbances are well-known consequences of the diabetic pathology. However, less is known about the cumulative effects of repeated changes in glycaemia, a characteristic of diabetes, on the electrolyte balance. We therefore investigated the ionic profiles of patients with type 1 diabetes during consecutive hyper- and/or hypoglycaemic events using the glucose clamp.

Methods In protocol 1, two successive hyperglycaemic excursions to $18 \mathrm{mmol} / 1$ were induced; in protocol 2, a hypoglycaemic excursion $(2.5 \mathrm{mmol} / \mathrm{l})$ was followed by a hyperglycaemic excursion $(12 \mathrm{mmol} / \mathrm{l})$ and another hypoglycaemic episode $(3.0 \mathrm{mmol} / \mathrm{l})$.

Results Blood osmolarity increased during hyperglycaemia and was unaffected by hypoglycaemia. Hyperglycaemia
\end{abstract}

Electronic supplementary material The online version of this article (doi:10.1007/s00125-011-2210-9) contains peer-reviewed but unedited supplementary material, which is available to authorised users.

A. Caduff $(\bowtie) \cdot$ G. Di Benedetto $\cdot$ M. S. Talary $\cdot$ S. Theander

Solianis Monitoring AG,

Leutschenbachstrasse 46,

CH-8050, Zürich, Switzerland

e-mail: andreas.caduff@solianis.com

H. U. Lutz

Institute of Biochemistry, ETH Zürich,

Zürich, Switzerland

L. Heinemann

Profil Institut fuer Stoffwechselforschung,

Neuss, Germany

G. Di Benedetto

Department of Mechanics, Politecnico di Torino,

Turin, Italy induced decreases in plasma $\mathrm{Na}^{+} \mathrm{Cl}^{-}$and $\mathrm{Ca}^{2+}$ concentrations and increases in $\mathrm{K}^{+}$concentrations. These changes were faithfully reproduced during a second hyperglycaemia. Hypoglycaemia provoked rapid and rapidly reversible increases in $\mathrm{Na}^{+}, \mathrm{Cl}^{-}$and $\mathrm{Ca}^{2+}$. In sharp contrast, $\mathrm{K}^{+}$levels displayed a rapid and substantial fall from which they did not fully recover even $2 \mathrm{~h}$ after the re-establishment of euglycaemia. A second hypoglycaemia caused an additional fall.

Conclusions/interpretation Repeated hyperglycaemia events do not lead to any cumulative effects on blood electrolytes. However, repeated hypoglycaemias are cumulative with respect to $\mathrm{K}^{+}$levels due to a very slow recovery following hypoglycaemia. These results suggest that recurring hypoglycaemic events may lead to progressively lower $\mathrm{K}^{+}$levels despite rapid re-establishment of euglycaemia. This warrants close monitoring of plasma $\mathrm{K}^{+}$levels combined with continuous glucose monitoring particularly in patients under intensive insulin therapy who are subject to repeated hypoglycaemic episodes.

Trial registration: Clinicaltrial.gov NCT01060917. Funding: Pendragon Medical AG, Solianis Monitoring AG

Keywords Blood - Cardiac arrhythmia . Dead in bed syndrome - Diabetes · Electrolytes · Glucose monitoring · Hyperglycaemia $\cdot$ Hypoglycaemia $\cdot$ Hypokalaemia $\cdot$ Insulin therapy

\section{Introduction}

Diabetes is characterised by impaired glucose homeostasis, mainly due to compromised insulin production and, in patients with type 2 diabetes, also to tissue insulin 
resistance. The principal manifestation of diabetes is chronically elevated blood glucose levels, i.e. hyperglycaemia. Long-term hyperglycaemia leads to microvascular damage; therefore a primary objective of diabetes treatment is to keep blood glucose levels within an acceptable range [1]. However, more acute threats to patients with diabetes are episodes of hypoglycaemia, which are often induced by glucose-lowering therapy [2]. Both hyper- and hypoglycaemia are associated with alterations of the electrolyte balance. For hyperglycaemia it is well known that in its severe manifestations such as diabetic ketoacidosis (DKA) and the hyperglycaemic, hyperosmolar state (HHS), profound changes occur in electrolyte balance, including hyperosmolarity and hyponatraemia [3, 4].

With respect to hypoglycaemia, early studies on the effects of insulin injections in dogs demonstrated a decrease in plasma $\mathrm{K}^{+}$levels [5]. In fact, hypokalaemia is now a well-established consequence of insulin-induced hypoglycaemia and is caused both by insulin itself and the counter-regulatory hormone adrenaline (epinephrine), secreted in response to hypoglycaemia $[6,7]$. Experimental work on many different tissues in vitro has shown that insulin has a stimulating effect on the $\mathrm{Na}^{+} / \mathrm{K}^{+}$pump [8-10]. In vivo, in studies in humans, this stimulatory effect on the $\mathrm{Na}^{+} / \mathrm{K}^{+}$pump is active at baseline insulin levels [11], and has been shown to be the cause of insulin-induced decreases in plasma $\mathrm{K}^{+}$after local perfusion of the forearm with insulin $[12,13]$. The stimulatory effect of insulin on the $\mathrm{Na}^{+} / \mathrm{K}^{+}$pump is also widely believed to underlie the hypoglycaemia-induced hypokalaemia [6, 7].

Studies of plasma $\mathrm{K}^{+}$levels and of other ion concentrations in blood using the hypoglycaemic glucose clamp, enabling tight control and rapid manipulation of glycaemia, are of interest but appear to be lacking in the literature.

Here, we present the first simultaneous measurements of all major ions and osmolarity in the blood of type 1 diabetes patients in response to in vivo hyper- and hypoglycaemic stimulation, using the glucose clamp technique. Our principal objective was to investigate if repeated hyper- and/or hypoglycaemic glucose excursions have any cumulative or conditioning effects on electrolyte levels, or if the electrolyte levels are determined by the level of glycaemia irrespective of prior glucose levels. On the mechanistic side, a particular aim was to investigate whether or not the mechanisms causing electrolyte changes in hyperglycaemia are operating in reverse in hypoglycaemia.

\section{Methods}

\section{Study participants}

Fifteen patients with type 1 diabetes mellitus participated in this Good Clinical Practice Study (age $27 \pm 6$ years, average duration of diabetes was $10.2 \pm 7.2$ years, BMI $23.2 \pm 2.1 \mathrm{~kg} / \mathrm{m}^{2}, \mathrm{HbA}_{1 \mathrm{c}} 6.9 \pm 0.8 \%(52.0 \pm 6.0 \mathrm{mmol} / \mathrm{mol})$; mean \pm SD are given for all values). It was confirmed that these participants had normal clinical chemistry and negative hepatitis and HIV serology, no alcohol or drug abuse and had not donated blood in the last 3 months. The participants were studied over two study days with different glucose clamp protocols (see below). This was an open, single-centre study approved by the local ethics committee performed according to the principles of the Declaration of Helsinki. All participants signed an informed consent.

\section{Study procedures}

The participants were connected to a Biostator (MTB Medizintechnik, Ulm, Germany), via a double lumen catheter inserted into a dorsal hand vein or lateral wrist vein to allow continuous blood glucose monitoring. The hand was inserted into a cuff delivering warm air in order to allow access to arterialised venous blood. A second catheter was inserted into an antecubital vein of the same arm for blood sampling. A third catheter was placed in the contralateral arm for glucose infusion (20\% in aqueous solution) and insulin solution (100 ml $150 \mathrm{mmol} / \mathrm{l}$ saline with $2 \mathrm{ml}$ of the participant's blood and $20 \mathrm{U}$ insulin).

Throughout the two protocols changes in the blood ion concentrations of sodium $\left(\mathrm{Na}^{+}\right)$, potassium $\left(\mathrm{K}^{+}\right)$, chloride $\left(\mathrm{Cl}^{-}\right)$and calcium $\left(\mathrm{Ca}^{2+}\right)$, osmolarity, as well as blood $\mathrm{pH}$, lactate (Lac), arterial oxygen pressure $\left(\mathrm{pO}_{2}\right)$, standard bicarbonate $\left(\mathrm{HCO}_{3}{ }^{-}\right)$and carbon dioxide pressure $\left(\mathrm{pCO}_{2}\right)$ were regularly measured using a blood gas/electrolyte analyser (ABL 700 radiometer, Copenhagen, Denmark). The blood glucose concentration was immediately measured in each blood sample using a laboratory glucose-oxidase method (Super GL Ambulance Glucose Analyzer, Hitado, Delecke-Möhnesee, Germany; $\mathrm{CV}<2.5 \%$ ). For measurements of ion concentrations and osmolarity, a total of up to $215 \mathrm{ml}$ blood was drawn for blood sampling. Every sample contained $3 \mathrm{ml}$ blood. A sample was taken every $5 \mathrm{~min}$ during the initial baseline period and during the phases of increase and decrease of blood glucose, and every $10 \mathrm{~min}$ during plateau phases. The ion and osmolarity measurements were performed immediately on the whole blood samples according to the guidelines for the ABL 700 radiometer.

\section{Study design}

Protocol 1 Two hyperglycaemic excursions were applied, each of duration $\sim 2 \mathrm{~h}$, interspersed by a return to baseline for $1 \mathrm{~h}$.

During the run-in phase of $120 \mathrm{~min}$, blood glucose was stabilised by intravenous infusion of insulin and/or glucose 
at $6.0 \mathrm{mmol} / \mathrm{l}$. At the beginning of the hyperglycaemic periods the insulin infusion was stopped. At the end of both hyperglycaemic periods the decline in blood glucose to a euglycaemic value of $6.0 \mathrm{mmol} / 1$ was induced by re-starting the intravenous insulin infusion. On average, a total of $45 \pm$ 27 (mean \pm SD) $\mathrm{U}$ insulin were infused for protocol 1 . Blood glucose was kept constant at this level after the second period for a further $1.5 \mathrm{~h}$.

Protocol 2 A hypoglycaemic event of $\sim 2 \mathrm{~h}$ was applied followed by a return to baseline, after which a hyperglycaemic challenge $(\sim 1.5 \mathrm{~h})$ was immediately followed by a hypoglycaemic event of the same magnitude and duration as the first challenge.

During the run-in phase of $120 \mathrm{~min}$, blood glucose was stabilised by intravenous infusion of insulin and/or glucose solution at $5.5 \mathrm{mmol} / \mathrm{l}$. Thereafter, blood glucose was lowered by intravenous administration of insulin to a hypoglycaemic level of $2.5 \mathrm{mmol} / 1$ for $30 \mathrm{~min}$. After an increase in glycaemia to a hyperglycaemic level of $11.1 \mathrm{mmol} / 1$ for $60 \mathrm{~min}$, an intravenous insulin infusion was started and again glycaemia was lowered to $2.5 \mathrm{mmol} / \mathrm{l}$. After $1 \mathrm{~h}$ at this level, blood glucose was elevated to an euglycaemic level of $5.5 \mathrm{mmol} / 1$ by means of an intravenous glucose infusion and kept constant at this level for another $1.5 \mathrm{~h}$. On average, a total of $49 \pm 29$ (mean $\pm \mathrm{SD}) \mathrm{U}$ insulin were infused for protocol 2 .

\section{Statistical analysis}

For each study protocol, the Student's $t$ test was for paired data: inside each group, a baseline glucose phase (corresponding to the first bar depicted in Fig. 1) was defined, and for each sample time point the hypothesis that the average value of each measured quantity of all participants are different from the baseline value was tested with a two tailed Student's test. On the basis of the $p$ value response $(p<0.05, p<0.01$ and $p<0.001)$, coloured horizontal bars in the figures indicate when, where and how the signals change with respect to the baseline values. Even though several comparisons between the baseline data and all the other time points were performed, corrections for multiple testing were not applied, for two main reasons:

At each time point in the different testing days, the measuring conditions (i.e. the experimental glucose and
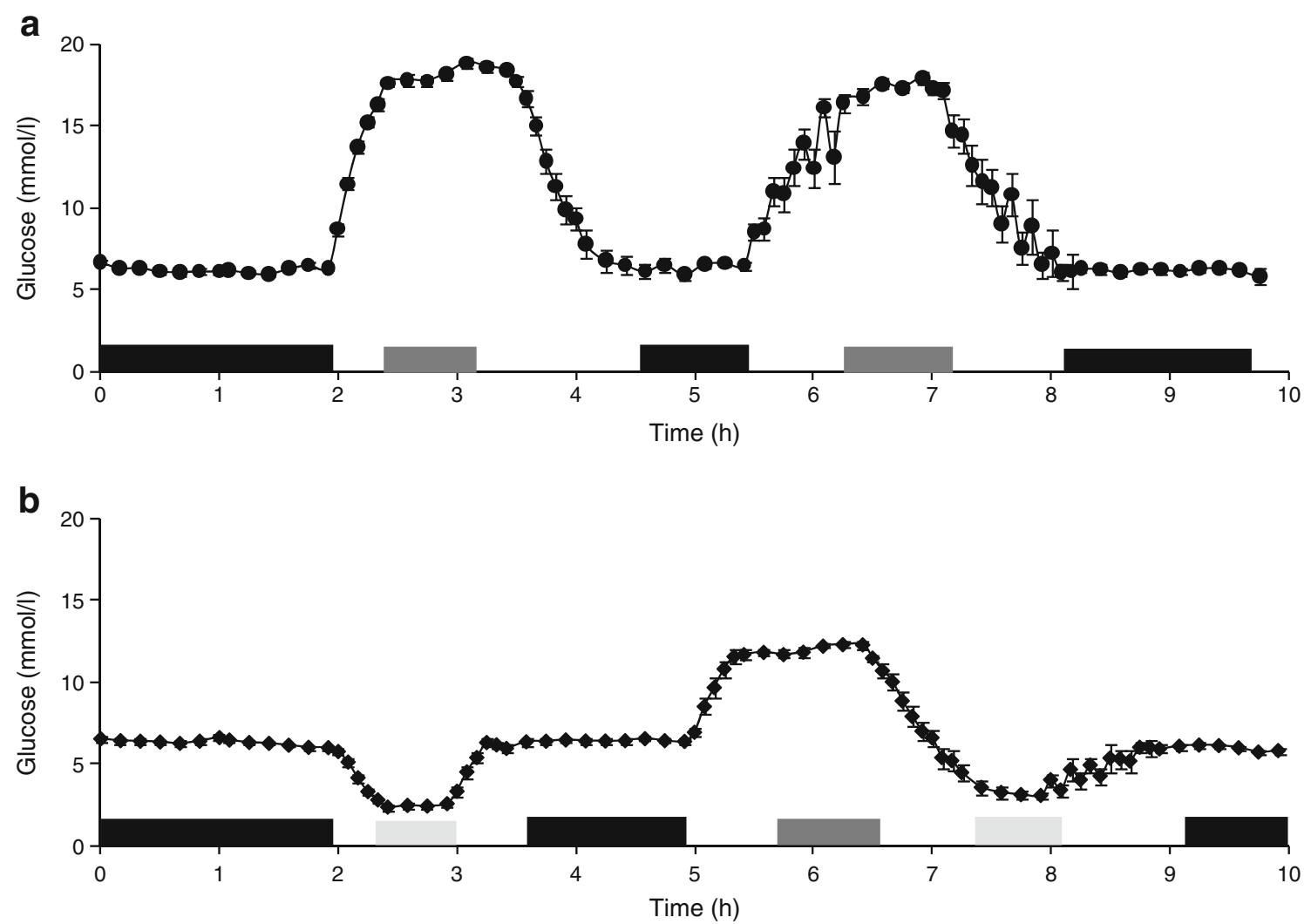

Fig. 1 a Blood glucose changes during protocol 1. The horizontal bars represent time periods with minimal changes in blood glucose. The averaged blood glucose and ion levels during these time periods are given in Table 1. b Blood glucose changes during protocol 2. Data representation as in (a). The averaged blood glucose and ion levels during the indicated periods are given in Table 2. Horizontal bars in light grey represent the time where there is a statistical significance of $p<0.05$, grey is $p 0.01$ and black is $p<0.01$ 
electrolytes values) are different, so we do not have a dataset that repeats several times with the same conditions for comparison. During the individual plateau phases we can consider that measuring conditions remain stable, but each plateau does not last for more than six sample points. In these phases the $p$ values are actually $<0.001$. These $p$ values indicate that we avoid the risk of obtaining fake test results.

The problem of multiple comparisons is present if there are many repetitions of the same test and some of the test results are significant, whereas others are not. In our analysis there is a quite complete correspondence between different phases (hyperglycaemic, euglycaemic and hypoglycaemic) and significant $p$ values, and these results could not be explained as being due to the added complexity of a multiple comparison result.

A statistical Student's $t$ test was also used to understand if there is any carry-over effect, i.e. if a first hyper- or hypoglycaemic stimulation has an impact on the second (electronic supplementary material [ESM] Methods). For each study protocol, the averaged values of each measured quantity (ion concentrations and osmolarity) during the different plateau phases of glycaemia (defined by the bars depicted in Fig. 1) are pair-wise compared against each other for statistical significance, using a two tailed Student's test, and the different periods are considered significantly different if $p<0.001$.

\section{Results}

\section{Glycaemic excursions}

Figure 1 shows the blood glucose profiles during protocol 1 (Fig. 1a) and protocol 2 (Fig. 1b). In both protocols we identified those time periods during which glucose levels changed minimally and averaged the measured quantities (ion concentrations, osmolarity and acid-base variables) during these periods with the aim of obtaining representative values for each period. The periods are indicated by the horizontal bars in Fig. 1a, b. The averaged values appear in Table 1 for protocol 1 and in Table 2 for protocol 2.

Sodium and chloride

In protocol 1 , the $\mathrm{Na}^{+}$concentrations decrease in a fully reversible manner in response to both hyperglycaemic excursions (Fig. 2a). Dynamically, the changes in $\mathrm{Na}^{+}$ concentration appear to be a near-perfect mirror image of the blood glucose levels in both hyperglycaemic excursions. Furthermore, the first excursion does not appear to have any conditioning effect on the second excursion. In protocol 2, blood $\mathrm{Na}^{+}$concentrations changed in the direction opposite to blood glucose changes without delays or transient effects. Thus, changes in the $\mathrm{Na}^{+}$concentrations reflect blood glucose excursions closely, during both hypoand hyperglycaemia.

The blood $\mathrm{Cl}^{-}$concentrations displayed a very similar response pattern to that of the $\mathrm{Na}^{+}$concentrations (Fig. 3a, b) and the changes were also quantificatively similar (Tables 1 and 2).

\section{Potassium}

The blood $\mathrm{K}^{+}$concentrations increased slowly in response to the two hyperglycaemic periods in protocol 1 (Fig. 4a). Indeed, the duration of the hyperglycaemic challenges were clearly too short to reach a new plateau level in $\mathrm{K}^{+}$ concentrations. In clear contrast to this slow response to the rapid increase in glycaemia, the drop in $\mathrm{K}^{+}$concentrations, once the hyperglycaemic periods ended, was fast and continued to hypokalaemic levels, significantly $(p<0.001)$ lower than the baseline values. A fast drop in the $\mathrm{K}^{+}$ concentrations was also observed as a response to the hypoglycaemia in protocol 2 (Fig. 4b), whereas the recovery during euglycaemia and subsequent hyperglycaemia was very slow. Indeed, at no subsequent time point after initiation of hypoglycaemia, including the subsequent hyperglycaemic challenge, did $\mathrm{K}^{+}$levels reach initial
Table 1 Averaged blood glucose and ion levels during selected time periods (horizontal bars, Fig. 1a) from protocol 1
$\mathrm{Eu}$, euglycaemia; Hyper, hyperglycaemia; Lac, lactate; Osm, osmolarity

\begin{tabular}{lrrrrr}
\hline Variable (mmol/l) & \multicolumn{1}{l}{$\begin{array}{l}\text { Baseline } \\
\text { Mean } \pm \text { SEM }\end{array}$} & $\begin{array}{l}\text { Hyper 1 } \\
\text { Mean } \pm \text { SEM }\end{array}$ & $\begin{array}{l}\text { Eu 1 } \\
\text { Mean } \pm \text { SEM }\end{array}$ & $\begin{array}{l}\text { Hyper 2 } \\
\text { Mean } \pm \text { SEM }\end{array}$ & \multicolumn{1}{l}{$\begin{array}{l}\text { Eu 2 } \\
\text { Mean } \pm \text { SEM }\end{array}$} \\
\hline Glucose & $6.22 \pm 0.05$ & $18.27 \pm 0.14$ & $6.36 \pm 0.16$ & $17.34 \pm 0.17$ & $6.10 \pm 0.07$ \\
$\mathrm{Na}^{+}$ & $138.88 \pm 0.10$ & $134.18 \pm 0.14$ & $139.08 \pm 0.16$ & $134.84 \pm 0.17$ & $139.20 \pm 0.13$ \\
$\mathrm{Cl}^{-}$ & $108.18 \pm 0.17$ & $105.23 \pm 0.28$ & $109.05 \pm 0.28$ & $106.00 \pm 0.31$ & $109.56 \pm 0.22$ \\
$\mathrm{~K}^{+}$ & $3.77 \pm 0.01$ & $4.03 \pm 0.02$ & $3.36 \pm 0.03$ & $3.87 \pm 0.04$ & $3.41 \pm 0.02$ \\
$\mathrm{Ca}^{2+}$ & $1.21 \pm 0.00$ & $1.17 \pm 0.00$ & $1.21 \pm 0.00$ & $1.17 \pm 0.00$ & $1.19 \pm 0.00$ \\
$\mathrm{Lac}$ & $0.88 \pm 0.03$ & $1.11 \pm 0.05$ & $1.73 \pm 0.04$ & $1.27 \pm 0.05$ & $1.68 \pm 0.04$ \\
Osm & $283.93 \pm 0.20$ & $286.58 \pm 0.29$ & $284.54 \pm 0.35$ & $287.07 \pm 0.29$ & $284.58 \pm 0.24$ \\
\hline
\end{tabular}


Table 2 Averaged blood glucose and ion levels during the selected time periods (horizontal bars, Fig. 1b) from protocol 2

\begin{tabular}{|c|c|c|c|c|c|c|}
\hline Variable $(\mathrm{mmol} / \mathrm{l})$ & $\begin{array}{l}\text { Baseline } \\
\text { Mean } \pm \text { SEM }\end{array}$ & $\begin{array}{l}\text { Hypo } 1 \\
\text { Mean } \pm \text { SEM }\end{array}$ & $\begin{array}{l}\text { Eu } 1 \\
\text { Mean } \pm \text { SEM }\end{array}$ & $\begin{array}{l}\text { Hyper } \\
\text { Mean } \pm \text { SEM }\end{array}$ & $\begin{array}{l}\text { Hypo } 2 \\
\text { Mean } \pm \text { SEM }\end{array}$ & $\begin{array}{l}\text { Eu } 2 \\
\text { Mean } \pm \text { SEM }\end{array}$ \\
\hline Glucose & $6.28 \pm 0.04$ & $2.45 \pm 0.07$ & $6.38 \pm 0.06$ & $12.25 \pm 0.11$ & $3.09 \pm 0.15$ & $6.03 \pm 0.08$ \\
\hline $\mathrm{Na}^{+}$ & $138.50 \pm 0.10$ & $140.65 \pm 0.17$ & $138.55 \pm 0.12$ & $136.74 \pm 0.27$ & $140.56 \pm 0.24$ & $138.59 \pm 0.16$ \\
\hline $\mathrm{Cl}^{-}$ & $108.57 \pm 0.17$ & $109.90 \pm 0.25$ & $108.53 \pm 0.16$ & $108.02 \pm 0.33$ & $111.37 \pm 0.41$ & $109.16 \pm 0.24$ \\
\hline $\mathrm{K}^{+}$ & $3.75 \pm 0.01$ & $3.26^{\mathrm{a}} \pm 0.02$ & $3.47^{\mathrm{a}} \pm 0.02$ & $3.65^{\mathrm{a}} \pm 0.04$ & $3.01^{\mathrm{a}, \mathrm{b}} \pm 0.03$ & $3.28^{\mathrm{a}, \mathrm{b}} \pm 0.03$ \\
\hline $\mathrm{Ca}^{2+}$ & $1.21 \pm 0.00$ & $1.23 \pm 0.00$ & $1.18 \pm 0.00$ & $1.16 \pm 0.01$ & $1.21 \pm 0.00$ & $1.19 \pm 0.00$ \\
\hline $\mathrm{Lac}$ & $0.80 \pm 0.01$ & $1.55 \pm 0.03$ & $1.13 \pm 0.03$ & $0.95 \pm 0.05$ & $1.64 \pm 0.05$ & $1.39 \pm 0.02$ \\
\hline Osm & $282.33 \pm 0.18$ & $283.70 \pm 0.31$ & $283.54 \pm 0.22$ & $285.82 \pm 0.55$ & $284.19 \pm 0.44$ & $283.16 \pm 0.32$ \\
\hline
\end{tabular}

For clarity, only those statistical comparisons mentioned in the text are indicated

$\mathrm{Eu}$, euglycaemia; Hyper, hyperglycaemia; Hypo, hypoglycaemia; Lac, lactate; Osm, osmolarity

${ }^{\mathrm{a}} p<0.001$ with respect to baseline; ${ }^{\mathrm{b}} p<0.001$ with respect to hypo 1

baseline levels. It is also noteworthy that the $\mathrm{K}^{+}$levels reached after the second hypoglycaemic challenge are substantially lower $(p<0.001$, Table 2$)$ than those maintained during the first hypoglycaemic challenge, suggesting a cumulative effect.

\section{Calcium}

The changes in blood $\mathrm{Ca}^{2+}$ concentrations mirror, in the same way as the changes in $\mathrm{Na}^{+}$, the dynamics of blood glucose in protocol 1 (Fig. 5a). Again, no conditioning
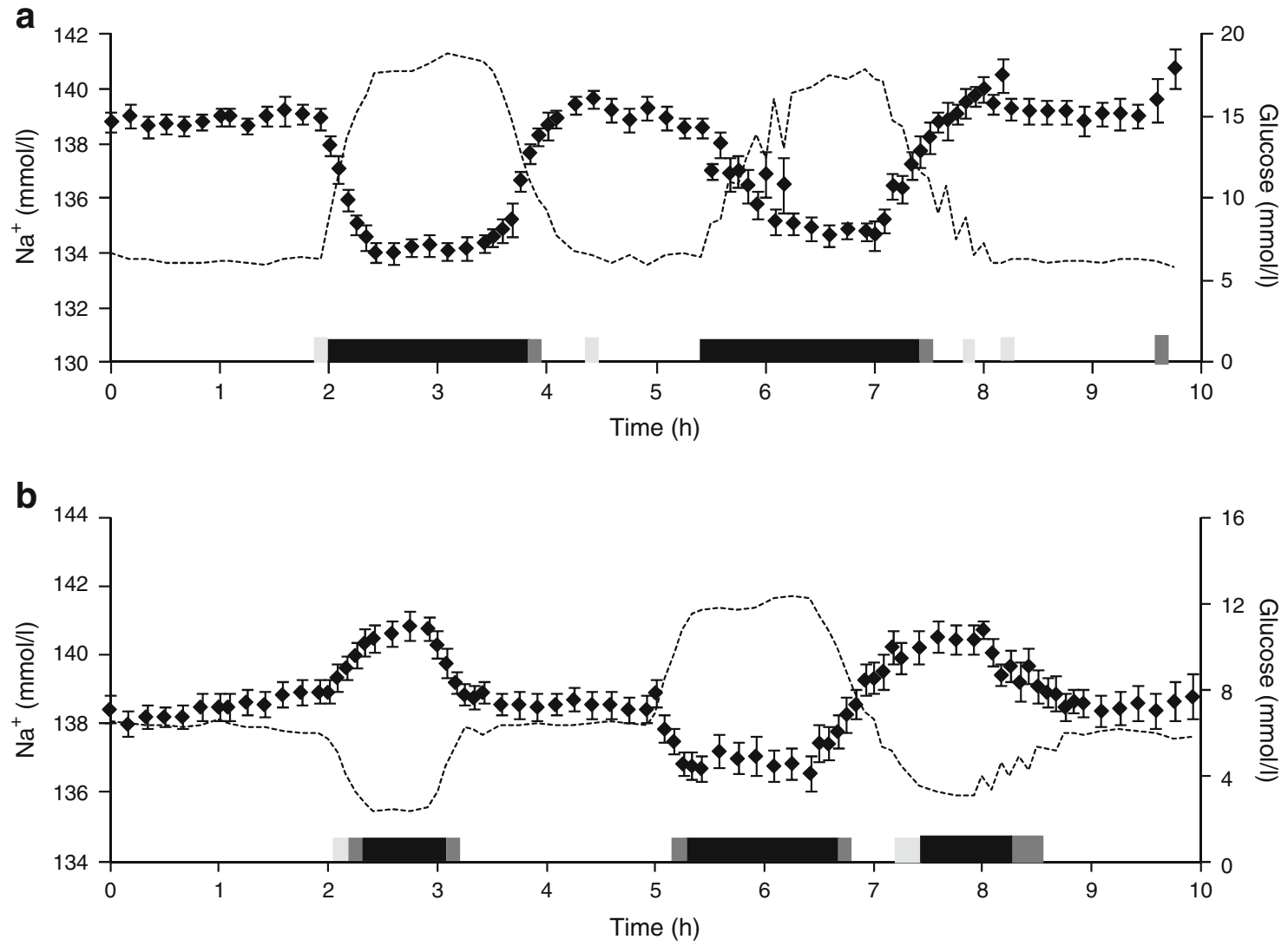

Fig. 2 Changes in blood $\mathrm{Na}^{+}$levels during protocol 1 (a) and protocol 2 (b). There were significant differences between $\mathrm{Na}^{+}$levels at a given time point compared with those during the first period of minimally changing glucose values as indicated by the first horizontal bars in Fig. 1a, b. Dotted line, blood glucose level. Horizontal bars in light grey represent the time where there is a statistical significance of $p<0.05$, grey is $p<0.01$ and black is $p<0.001$ 

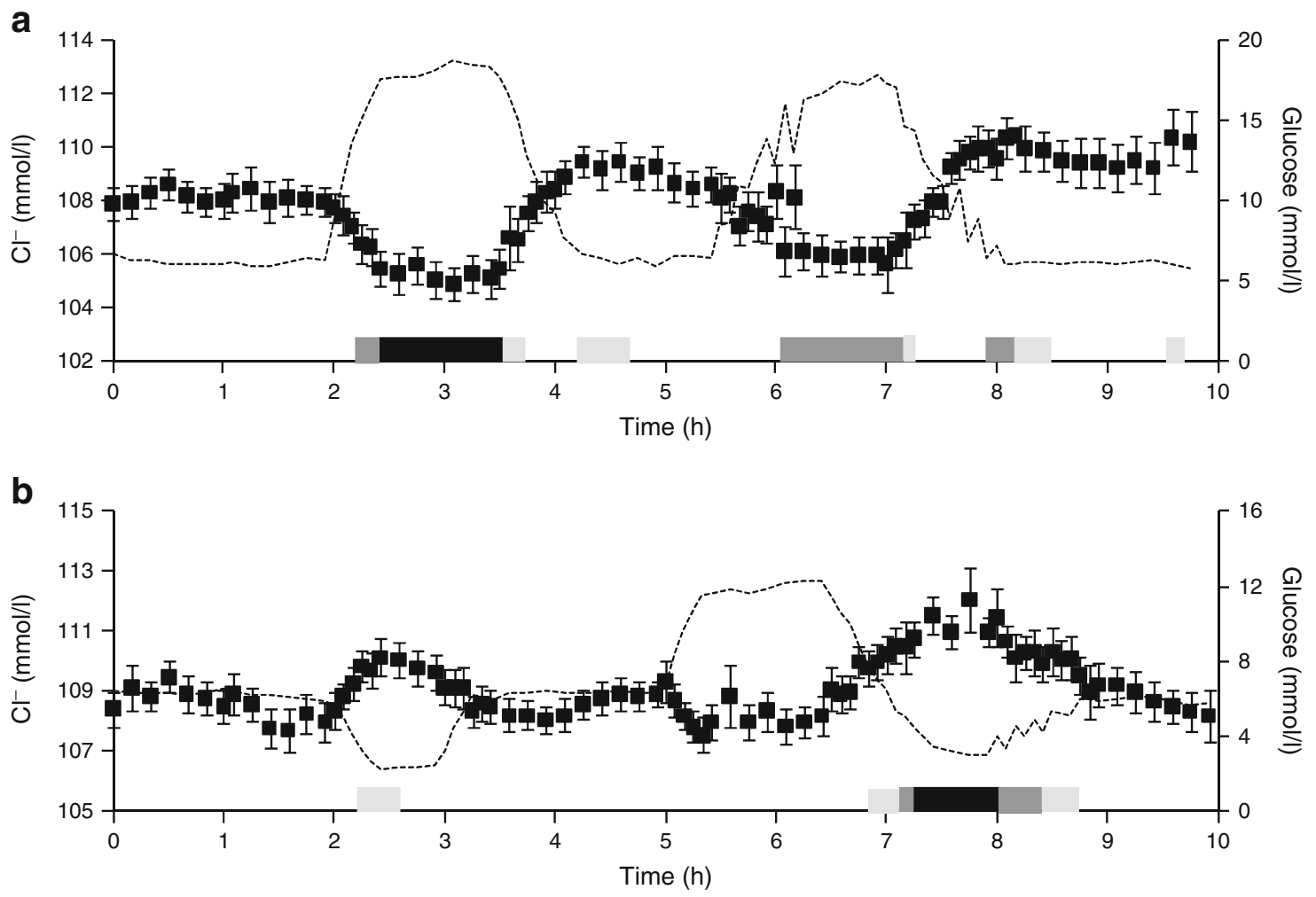

Fig. 3 Changes in blood $\mathrm{Cl}^{-}$levels during protocol 1 (a) and protocol 2 (b). Data presentation as in Fig. 2. Dotted line, blood glucose level. Horizontal bars in light grey represent the time where there is a statistical significance of $p<0.05$, grey is $p<0.01$ and black is $p<0.001$
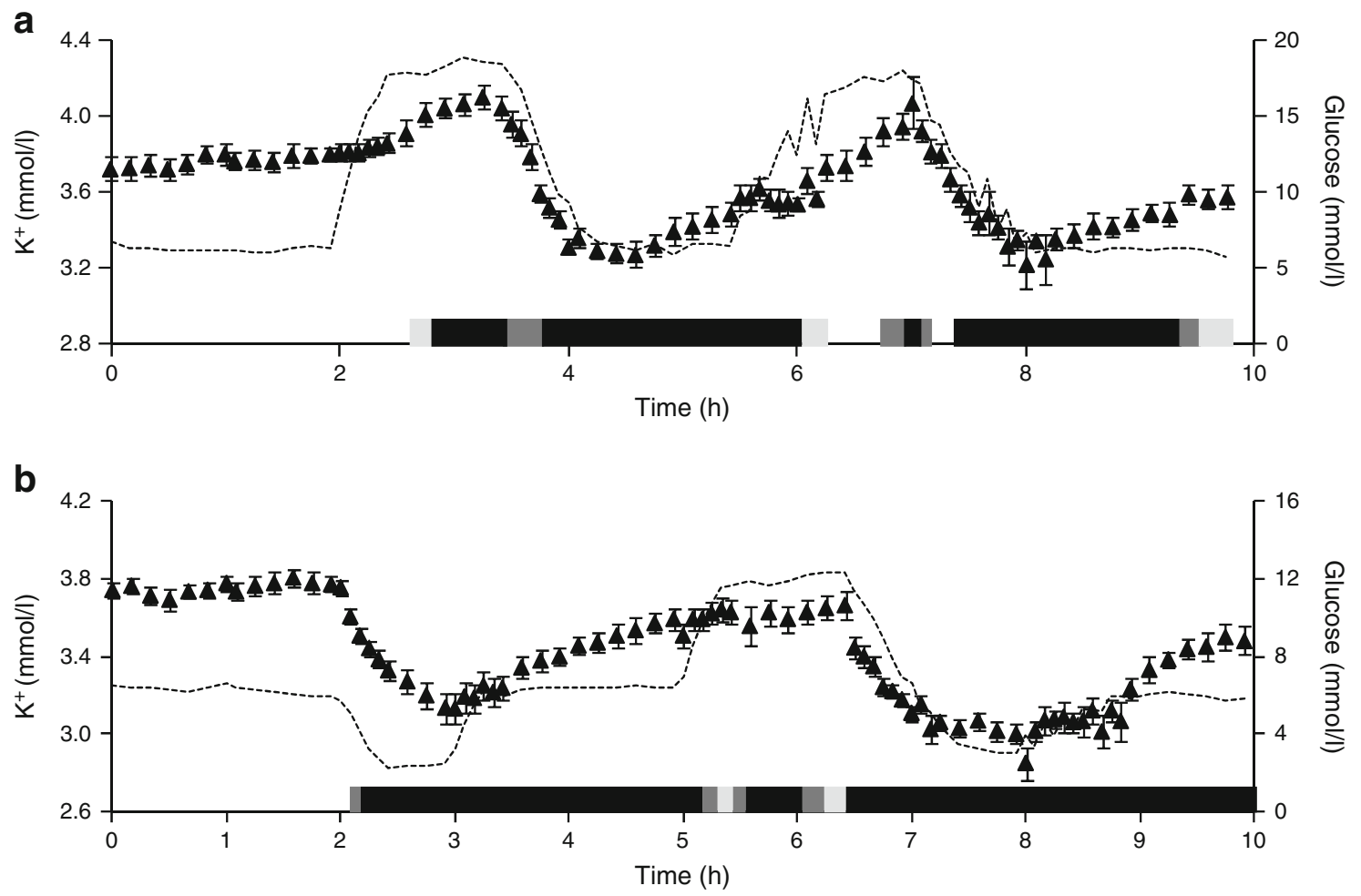

Fig. 4 Changes in blood $\mathrm{K}^{+}$levels during protocol 1 (a) and protocol 2 (b). Data presentation as in Fig. 2. Dotted line, blood glucose level. Horizontal bars in light grey represent the time where there is a statistical significance of $p<0.05$, grey is $p<0.01$ and black is $p<0.001$ 

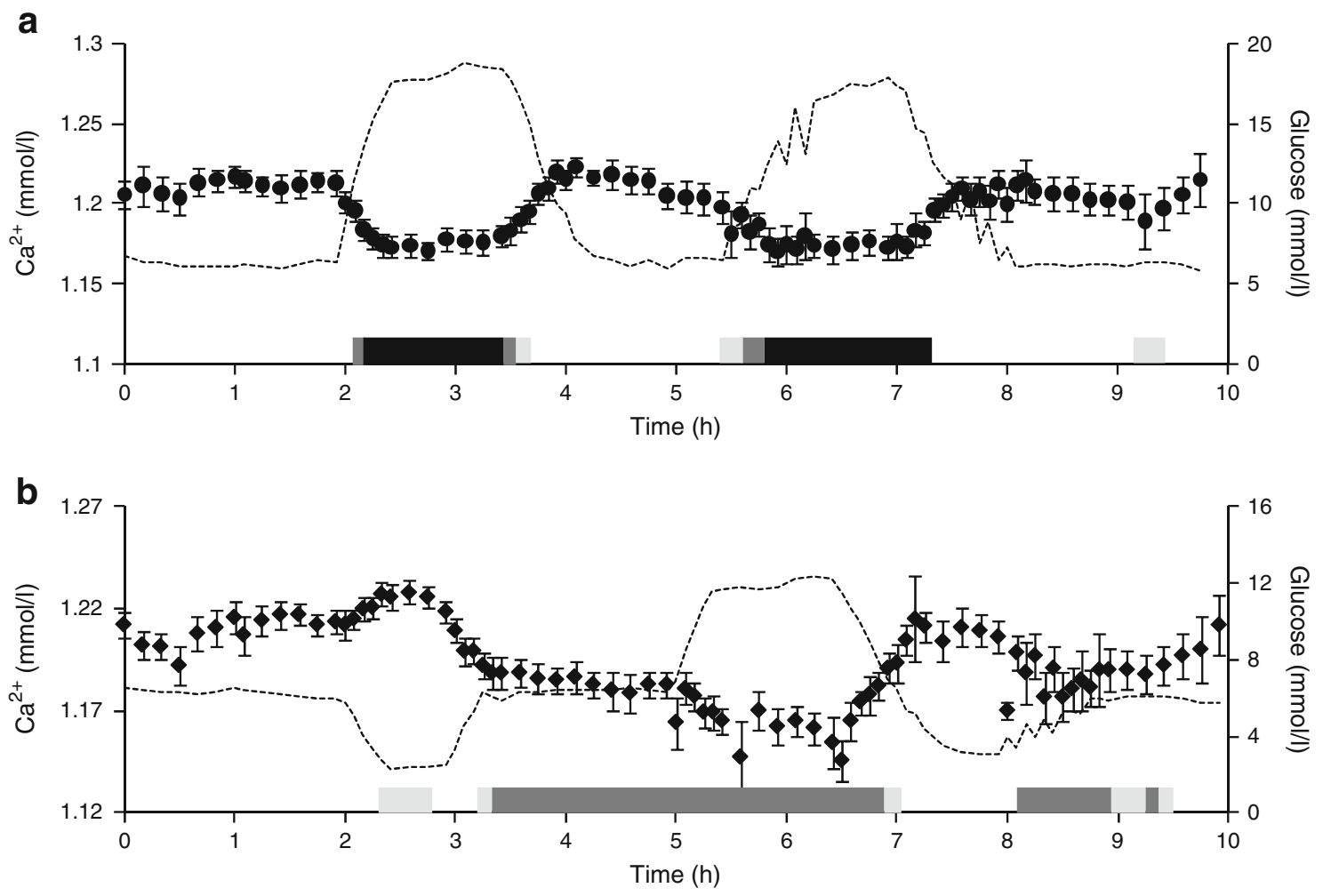

Fig. 5 Changes in blood $\mathrm{Ca}^{2+}$ levels during protocol 1 (a) and protocol 2 (b). Data presentation as in Fig. 2. Dotted line, blood glucose level. Horizontal bars in light grey represent the time where there is a statistical significance of $p<0.05$, grey is $p<0.01$ and black is $p<0.001$

effects of the first hyperglycaemic period on the second period were prominent. The responses to hypoglycaemia are also, qualitatively, very similar to those of the $\mathrm{Na}^{+}$ levels (Fig. 5a).

\section{Lactate and acid-base variables}

No physiologically relevant changes in blood $\mathrm{pH}$, bicarbonate or $\mathrm{pCO}_{2}$ concentrations were observed during either protocol 1 or 2 (data not shown). In contrast, blood lactate concentrations started to slowly and very moderately increase during increases in glycaemia, whereas decreases in glycaemia led to large and rapid increases in lactate levels. This response pattern is similar in both protocols (Fig. 6).

\section{Osmolarity}

Plasma osmolarity increases in response to both hyperglycaemic periods in protocol 1 (Fig. 7a). No change was, however, observed when blood glucose decreased from euglycaemia to hypoglycaemia in protocol 2 . Nevertheless, when starting from hyperglycaemia and going down to hypoglycaemia, a small decrease in osmolarity was observed (Fig. 7b). As an internal control of our measurements we also calculated the osmolarity as the sum of $\mathrm{Na}^{+}, \mathrm{Cl}^{-}, \mathrm{K}^{+}, \mathrm{Ca}^{2+}$, lactate, glucose and bicarbonate concentrations. The results are shown in Fig. 7 as thick grey lines. It is seen that the calculated osmolarity follows the measured osmolarity very closely. In fact, at no time in either protocol was the difference larger than $0.9 \%$. This also indicates that no solute changes of a magnitude higher than $1-2 \mathrm{mmol} / \mathrm{l}$ took place in solutes other than those measured here.

\section{Discussion}

This study shows that plasma concentrations of $\mathrm{Na}^{+}, \mathrm{Cl}^{-}$and $\mathrm{Ca}^{2+}$ decrease in response to moderate hyperglycaemia in patients with type 1 diabetes. These changes take place without delays and are rapidly reversible. Furthermore, a preceding hyperglycaemic period had no conditioning effect on a second hyperglycaemic period. Thus, it appears as if hyperglycaemia-associated changes in these blood electrolyte ion concentrations have no long-term systemic effects, at least within the time periods studied, and are reversible.

The ionic changes occurring as a result of hypoglycaemia were all in the opposite direction to the changes following hyperglycaemia, with the exception of those of lactate, which increased both as a result of hyper- and hypoglycaemia. 

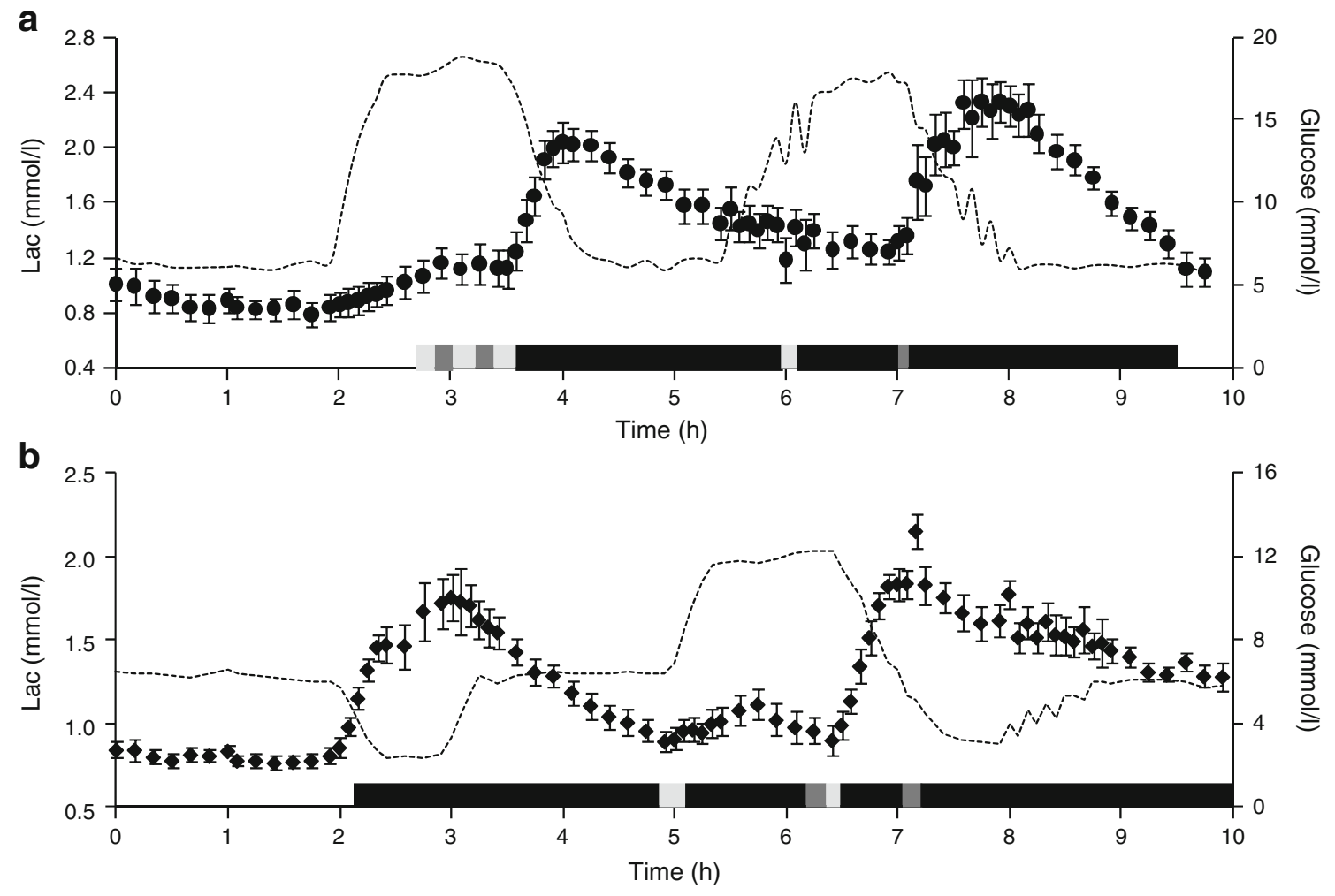

Fig. 6 Changes in lactate (Lac) levels during protocol 1 (b) and protocol 2 (b). Data presentation as in Fig. 2. Horizontal bars in light grey represent the time where there is a statistical significance of $p<0.05$, grey is $p 0.01$ and black is $p<0.01$
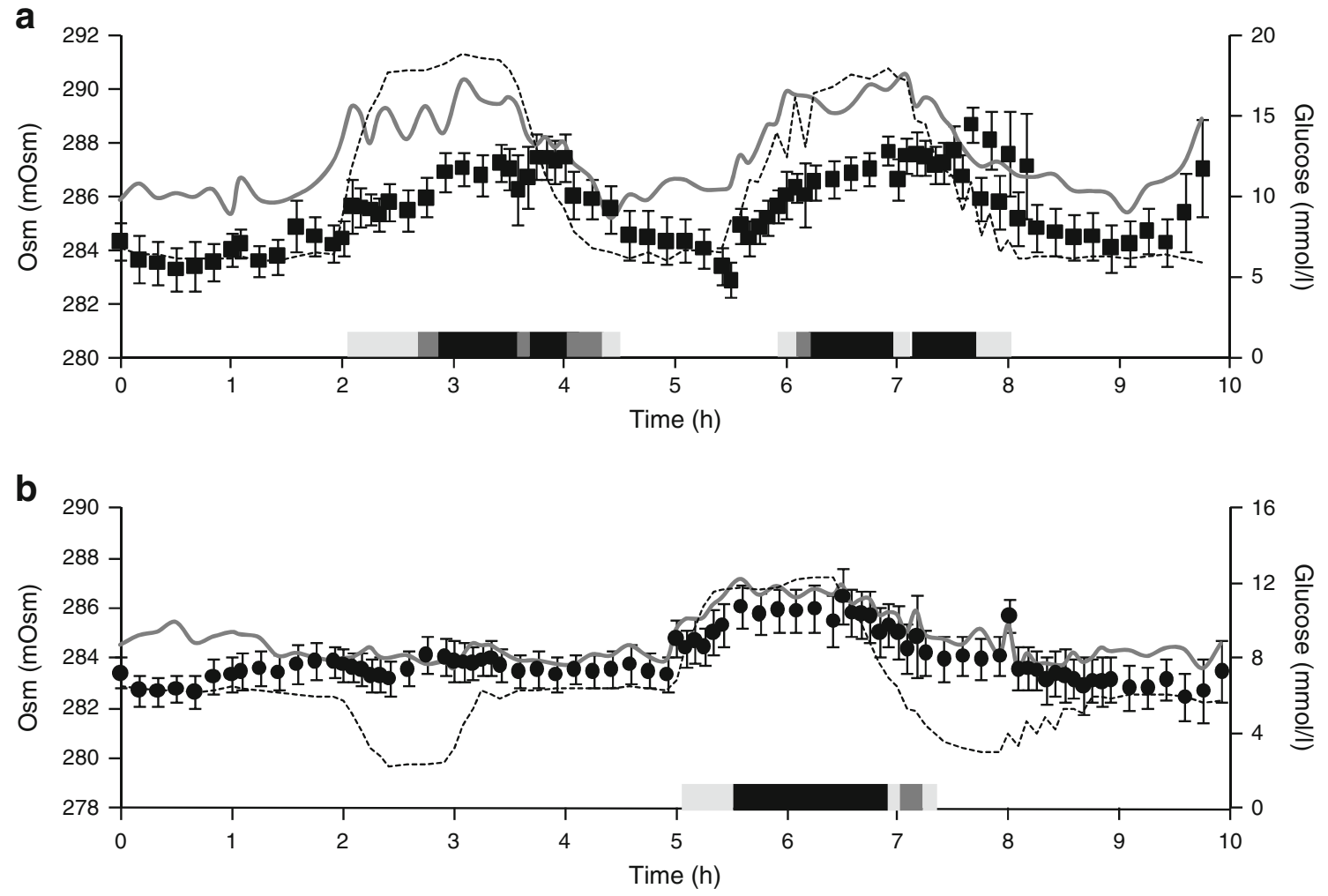

Fig. 7 Changes in blood osmolarity during protocol 1 (a) and protocol 2 (b). Data presentation as in Fig. 2. The thick grey line represents the calculated osmolarities (Osm) (see text). Horizontal bars in light grey represent the time where there is a statistical significance of $p<0.05$, grey is $p 0.01$ and black is $p<0.01$ 
Although the decrease in $\mathrm{K}^{+}$levels as a result of hypoglycaemia is a well-known phenomenon (reviewed in [6]), we have not found any information in controlled studies on humans regarding changes of $\mathrm{Na}^{+}, \mathrm{Cl}^{-}$or plasma osmolarity. In one study, increases in plasma $\mathrm{Ca}^{2+}$ levels have been reported and were attributed to increases in plasma calcitonin concentrations [14].

An important aim of this study was to elucidate if changes in plasma ion concentrations resulting from the induction of hypo- or hyperglycaemia involve similar mechanisms. One hypothesis for the mechanism in hyperglycaemia-induced hyponatraemia that has gained wide acceptance $[3,15,16]$ is that the increased osmolarity associated with elevated plasma glucose levels leads to an osmotically driven flux of water from cells into the interstitium and the blood, thus effectively diluting the ions in the blood. We find that such a dilution plausibly explains not only the drop in $\mathrm{Na}^{+}$levels but also the decreasing levels of $\mathrm{Cl}^{-}$during hyperglycaemia, whereas the increase in $\mathrm{K}^{+}$levels must have other causes (see below). It is tempting to assume that such a dilution mechanism could also operate in reverse mode in hypoglycaemia, such that a net flow of water from blood to cells would take place accompanied by an increase in the ion concentrations in the plasma. However, the fact that plasma osmolarity did not change during hypoglycaemia (Fig. 7) excludes the possibility of water flowing from blood to cells. The observed increases in plasma ion concentrations are therefore not caused by a concentration of the plasma, but by hypoglycaemia-induced ion translocations across plasma membranes. The nature and possible origins of these ion translocations are discussed below.

The hypokalaemia associated with insulin-induced hypoglycaemia is generally thought to be caused by stimulation of the $\mathrm{Na}^{+} / \mathrm{K}^{+}$pump $[6,7]$. Insulin plays an important part in ionic regulation by its stimulatory effect [11-13] on the $\mathrm{Na}^{+} / \mathrm{K}^{+}$pump. The increase in the $\mathrm{K}^{+}$levels during hyperglycaemia in protocol 1 may thus be explained by the withdrawal of that insulin, which was given to keep the study participants euglycaemic at baseline. The counterregulatory hormones adrenaline and noradrenaline (norepinephrine) also act in a stimulatory manner on the $\mathrm{Na}^{+} / \mathrm{K}^{+}$ pump [17, 18], and adrenaline has also been shown to contribute to hypoglycaemia-induced hypokalaemia [7]. The low $(2.83 \mathrm{mmol} / \mathrm{l})$ glucose threshold of noradrenaline release [19] makes noradrenaline unlikely to participate substantially in hypokalaemia, at least in asymptomatic moderate hypoglycaemias.

The insulin-induced stimulation of the $\mathrm{Na}^{+} / \mathrm{K}^{+}$pump readily explains the sharp drop in $\mathrm{K}^{+}$levels seen both at the onset of hypoglycaemia in protocol 2 and the termination of hyperglycaemia in both protocols. In hypoglycaemia, an adrenaline-induced stimulation of the $\mathrm{Na}^{+} / \mathrm{K}^{+}$pump may also contribute $[7,20]$. These results are also consistent with the fast action of insulin on $\mathrm{K}^{+}$levels reported by Petersen et al. [7].

Also the increase in the $\mathrm{Na}^{+}$levels can be explained by the effect of insulin on the $\mathrm{Na}^{+} / \mathrm{K}^{+}$pump. Furthermore, the stimulation of the electrogenic [9] $3 \mathrm{Na}^{+} / 2 \mathrm{~K}^{+}$pump leads to hyperpolarisation, which causes an outflux of $\mathrm{Cl}^{-}$, thus also explaining the changes seen in $\mathrm{Cl}^{-}$levels. It is true that insulin, albeit in euglycaemic conditions, has been shown to substantially decrease renal $\mathrm{Na}^{+}$excretion $(\sim 50 \%)$ and increase free water clearance by about $30 \%$ [21]. However, the slower dynamics of these effects and more importantly, as shown in the paper, that no effects were seen on the plasma $\mathrm{Na}^{+}$levels, may indicate that these effects are not responsible for the fast increase in $\mathrm{Na}^{+}$levels, which we observe upon insulin-induced hypoglycaemia.

The rapid decline in $\mathrm{K}^{+}$levels due to an insulin-induced hypoglycaemia (protocol 2) is in sharp contrast to its sluggish and incomplete recovery during a $2 \mathrm{~h}$ phase of euglycaemia. In fact, $\mathrm{K}^{+}$levels do not reach baseline values during euglycaemia nor during the subsequent hyperglycaemic period, which extended to $4.5 \mathrm{~h}$ after the termination of hypoglycaemia. During the second hypoglycaemic event, $\mathrm{K}^{+}$levels fall below the levels of the first, thus indicating that successive hypoglycaemias may be cumulative with respect to $\mathrm{K}^{+}$levels. It should be emphasised that this property of potassium ions is unique and irreversible, whereas changes of all other ions, except for lactate [22], were rapidly reversible.

Aldosterone plays an important role in controlling plasma $\mathrm{K}^{+}$levels by increasing renal $\mathrm{K}^{+}$excretion $[23,24]$. The levels of aldosterone are controlled both by the plasma $\mathrm{K}^{+}$ levels and by the renin-angiotensin system [23-25]. In hypoglycaemia, aldosterone levels increase due to the activation of the renin-angiotensin system [26, 27], and this increase appears to be an effect by hypoglycaemia and not of insulin, as euglycaemic hyperinsulinaemia leads to a decrease of aldosterone. This decrease was furthermore shown to be caused by falling $\mathrm{K}^{+}$levels, as infusion of $\mathrm{KCl}$ abolished the effect of insulin on aldosterone levels [28].

Aldosterone induces the formation of serum/glucocorticoid regulated kinase 1 (SGK-1), which regulates the potassium channel renal outer medulla potassium (ROMK) in the distal nephron $[25,29]$. The renal $\mathrm{K}^{+}$secretion is coupled to absorption of $\mathrm{Na}^{+}$via the apical $\mathrm{Na}^{+}$channel and basolateral $\mathrm{Na}^{+} / \mathrm{K}^{+}$pumps [30]. In consequence, an aldosterone-induced drop in plasma $\mathrm{K}^{+}$levels is coupled to an increase in $\mathrm{Na}^{+}$levels. Such coupling has also been demonstrated by direct aldosterone infusion in humans [31] and adrenolectomised dogs [32]. In view of our observation of a sustained suppression of the $\mathrm{K}^{+}$levels following hypoglycaemia and the absence of any corresponding longacting effect on the $\mathrm{Na}^{+}$levels, a mechanism other than that 
of aldosterone-induced changes must therefore be sought. Frindt et al. have demonstrated the existence of a pathway for renal $\mathrm{K}^{+}$secretion, which under certain circumstances may operate in parallel to the aldosterone-stimulated pathway. At physiological plasma concentrations of potassium (3.5-4.5 mmol/1), the driving force for the potassium secretion, the $\mathrm{Na}^{+}$entry via apical $\mathrm{Na}^{+}$channel, is fully inhibited by amiloride $(0.05 \mu \mathrm{mol} / \mathrm{min})$ [33]. If potassium feeding was increased by about 10 -fold overnight or for a week, the organism could largely maintain physiological plasma potassium concentrations, but a significant portion of this potassium secretion evidently bypassed the apical $\mathrm{Na}^{+}$channels, because plasma potassium concentrations were $5.5-8.9 \mathrm{mmol} / 1$ in the presence of amiloride, which exclusively inhibits the $\mathrm{Na}^{+}$channel-dependent portion [33]. The nature of the amiloride-insensitive excretion is so far unclear, but may involve an electroneutral excretion via a $\mathrm{KCl}$-cotransporter [33]. In view of the fact that no delay or irreversibility was seen in the $\mathrm{Na}^{+}$levels, we believe that the aldosterone-stimulated pathway is unlikely to cause the slow and incomplete recovery of $\mathrm{K}^{+}$levels. On the other hand, the amiloride-insensitive pathway described by Frindt et al., which is not associated with any changes in plasma $\mathrm{Na}^{+}$levels may well be involved in the suppression of plasma $\mathrm{K}^{+}$levels following hypoglycaemia.

Another mechanism that may specifically contribute to explain the very slow recovery of $\mathrm{K}^{+}$levels after an insulininduced hypoglycaemic challenge was demonstrated by DeFronzo et al. [34]. They showed that insulin leads to an initial rapid uptake of $\mathrm{K}^{+}$into the splanchnic bed (during the first hour of insulin administration), which reversed after the second hour, however, and turned into a slower leakage from the splanchnic bed into plasma. This reversal was shown to be dependent on the hypokalaemia, as it could be substantially attenuated by infusion of large amounts of $\mathrm{KCl}$. It was estimated that during the first hour, most $(\sim 70 \%)$ of the drop in plasma $\mathrm{K}^{+}$levels was due to splanchnic uptake, whereas from the second hour onwards, insulin-induced peripheral $\mathrm{K}^{+}$uptake dominates. Thus, the effects of insulin on plasma $\mathrm{K}^{+}$levels during the first hour were determined by escape into the splanchnic bed and only after the second hour did peripheral insulin-induced $\mathrm{K}^{+}$uptake predominate. Overall, this scheme with the splanchnic bed acting as a temporary $\mathrm{K}^{+}$buffer represents a delay of the effects of insulin on peripheral $\mathrm{K}^{+}$uptake, which might well explain the marked difference in dynamics of the $\mathrm{K}^{+}$levels being rapid at the onset of hypoglycaemia and very slow at its termination as observed in our measurements.

\section{Clinical implications}

The benefits of intensive control of glycaemia to prevent microvascular complications have been estab- lished in large long-term studies of patients with type 1 $[35,36]$, as well as type 2 , diabetes [37]. In patients with type 1 diabetes, a beneficial effect has also been demonstrated with respect to cardiovascular disease [38]. Although these studies were essentially performed on outpatients, other studies have shown that tight glycaemic control is beneficial also in critically ill patients, reducing the morbidity and mortality in surgical intensive care units [39] and morbidity in medical intensive care units [40].

A consistently recurring theme throughout these studies is, however, the increased risk of hypoglycaemia turning the benefit into a loss, if not adequately managed. In a recent study this was shown by an increased mortality if the glucose target was set at $4.5-6.0 \mathrm{mmol} / 1$ than when it was set at $10 \mathrm{mmol} / 1$ [41]. Intensive glycaemic control could, therefore, be a control of both the upper and the lower bounds of glycaemia, a task that puts increasing demands on the glucose monitoring and particularly on the frequency of blood glucose tests, with continuous monitoring [42] being the ideal solution.

Hypokalaemia, following insulin-induced hypoglycaemia, is well known for causing prolonged cardiac repolarisation, a phenomenon identified as a prolongation of the QT interval in the electrocardiogram, which may cause serious arrhythmias [6, 43]. Furthermore it has been demonstrated that the QTc interval also prolongs during euglycaemic hyperinsulinaemia, thus indicating that the effect is unrelated to the effects of insulin on glucose uptake but more likely to its effects on plasma $\mathrm{K}^{+}$levels [44]. Hypokalaemia has also been demonstrated to be a principal cause of prolongation of the QT interval in insulin-induced hypoglycaemia [45]. It has furthermore been discussed [6] that this effect on the heart may be the cause of sudden death, the dreaded 'Dead in Bed Syndrome', which occurs in type 1 diabetic patients [46] and has been linked to an intensive glycaemic control with insulin therapy [47]. The most pertinent finding with direct clinical implications in this study is therefore the slow recovery from hypokalaemia after hypoglycaemia. This effect causes repeated hypoglycaemias to be partially cumulative with respect to kalaemia and may lead to dangerously low $\mathrm{K}^{+}$levels even after the reestablishment of euglycaemia. It is likely that this cumulative effect is more pronounced when hypoglycaemic episodes are occurring within a short time span. Thus, further investigations should be performed also in critical care patients to evaluate whether the findings presented here are of clinical relevance. In the present work, the absence of insulin measurements is a weakness. Therefore, in future activities on this subject, insulin, as well as additional candidates, should be included in the list of measured variables such as $\mathrm{C}$-peptide or adrenaline. 
Acknowledgements The authors wish to thank F. Verrey (Institute of Physiology, University of Zurich, Zurich, Switzerland), P. Ambuehl (Nephrology Department, Waid Hospital, Zurich, Switzerland) and R. Slingerland (Clinical Chemistry Department), Isala klinieken, Zwolle, the Netherlands) for valuable discussions.

All authors have made significant contributions to the conception, design, or analysis and interpretation of presented data as well as in drafting the article or revising it critically for important intellectual content and gave their approval for the final version.

Duality of interest statement The authors declare that there is no duality of interest associated with this manuscript.

\section{References}

1. Nathan DM (1993) Long-term complications of diabetes mellitus. N Engl J Med 328:1676-1685

2. Cryer PE (2005) Mechanisms of hypoglycemia-associated autonomic failure and its component syndromes in diabetes. Diabetes 54:3592-3601

3. Kitabchi AE, Umpierrez GE, Murphy MB (2004) Diabetic ketoacidocis and hyperglycemic hyperosmolar state. In: Defronzo RA, Ferrannini E, Keen H, Zimmet P (eds) International textbook of diabetes mellitus, 3rd edn. Wiley, Chichester, pp 1101-1119

4. Kitabchi AE, Umpierrez GE, Miles JM, Fisher JN (2009) Hyperglycemic crises in adult patients with diabetes. Diabetes Care 32:1335-1343

5. Briggs AP, Koechig I, Doisy EA, Weber CJ (1924) Some changes in the composition of blood due to the injection of insulin. J Biol Chem 58:721-730

6. Heller SR, Robinson RT (2000) Hypoglycaemia and associated hypokalaemia in diabetes: mechanisms, clinical implications and prevention. Diabetes Obes Metab 2:75-82

7. Petersen KG, Schluter KJ, Kerp L (1982) Regulation of serum potassium during insulin-induced hypoglycemia. Diabetes 31:615-617

8. Clausen T, Kohn PG (1977) The effect of insulin on the transport of sodium and potassium in rat soleus muscle. J Physiol 265:19-42

9. Clausen $\mathrm{T}$ (2003) $\mathrm{Na}^{+}-\mathrm{K}^{+}$pump regulation and skeletal muscle contractility. Physiol Rev 83:1269-1324

10. Hansen PS, Buhagiar KA, Gray DF, Rasmussen HH (2000) Voltage-dependent stimulation of the $\mathrm{Na}(+)-\mathrm{K}(+)$ pump by insulin in rabbit cardiac myocytes. Am J Physiol Cell Physiol 278:C546C553

11. Defronzo RA, Sherwin RS, Dillingham M, Hendler R, Tamborlane WV, Felig P (1978) Influence of basal insulin and glucagon secretion on potassium and sodium metabolism. Studies with somatostatin in normal dogs and in normal and diabetic human beings. J Clin Invest 61:472-479

12. Andres R, Baltzan MA, Cader G, Zierler KL (1962) Effect of insulin on carbohydrate metabolism and on potassium in the forearm of man. J Clin Invest 41:108-115

13. Ferrannini E, Taddei S, Santoro D et al (1988) Independent stimulation of glucose metabolism and $\mathrm{Na}^{+}-\mathrm{K}^{+}$exchange by insulin in the human forearm. Am J Physiol 255:E953-E958

14. Body JJ, Cryer PE, Offord KP, Heath H III (1983) Epinephrine is a hypophosphatemic hormone in man. Physiological effects of circulating epinephrine on plasma calcium, magnesium, phosphorus, parathyroid hormone, and calcitonin. J Clin Invest 71:572-578

15. Hillier TA, Abbott RD, Barrett EJ (1999) Hyponatremia: evaluating the correction factor for hyperglycemia. Am J Med 106:399403

16. Rose BD, Post T (2001) Clinical physiology of acid-base and electrolyte disorders. McGraw-Hill, New York
17. Clausen T, Flatman JA (1980) Beta 2-adrenoceptors mediate the stimulating effect of adrenaline on active electrogenic $\mathrm{Na}-\mathrm{K}$ transport in rat soleus muscle. Br J Pharmacol 68:749-755

18. Clausen T, Flatman JA (1987) Effects of insulin and epinephrine on $\mathrm{Na}^{+}-\mathrm{K}^{+}$and glucose transport in soleus muscle. Am J Physiol 252:E492-E499

19. Schwartz NS, Clutter WE, Shah SD, Cryer PE (1987) Glycemic thresholds for activation of glucose counterregulatory systems are higher than the threshold for symptoms. J Clin Invest 79:777-781

20. De Feo P, Gallai V, Mazzotta G et al (1988) Modest decrements in plasma glucose concentration cause early impairment in cognitive function and later activation of glucose counterregulation in the absence of hypoglycemic symptoms in normal man. J Clin Invest 82:436-444

21. DeFronzo RA, Cooke CR, Andres R, Faloona GR, Davis PJ (1975) The effect of insulin on renal handling of sodium, potassium, calcium and phosphate in man. J Clin Invest 55:845855

22. Halperin ML, Fields AL (1985) Lactic acidosis - emphasis on the carbon precursors and buffering of the acid load. Am J Med Sci 289:154-159

23. Cooke CR, Horvath JS, Moore MA, Bledsoe T, Walker WG (1973) Modulation of plasma aldosterone concentration by plasma potassium in anephric man in the absence of a change in potassium balance. J Clin Invest 52:3028-3032

24. Himathongkam T, Dluhy RG, Williams GH (1975) Potassiumaldosterone-renin interrelationships. J Clin Endocrinol Metab 41:153-159

25. Wang WH, Giebisch G (2009) Regulation of potassium (K) handling in the renal collecting duct. Pflugers Arch 458:157-168

26. Hata S, Kunita H, Okamoto M (1976) Aldosterone response to hypoglycemia: evidence of ACTH mediation. J Clin Endocrinol Metab 43:173-177

27. Hochberg Z, Dickerman Z, Kaufman H, Laron Z (1980) Evaluation of the renin-aldosterone system during hypo- and hyperglycemia in children and adolescents. Horm Res 12:16-21

28. Ferrannini E, Galvan AQ, Santoro D, Natali A (1992) Potassium as a link between insulin and the renin-angiotensin-aldosterone system. J Hypertens Suppl 10:S5-S10

29. Verrey F, Loffing J, Zecevic M, Heitzmann D, Staub O (2003) SGK1: aldosterone-induced relay of $\mathrm{Na}^{+}$transport regulation in distal kidney nephron cells. Cell Physiol Biochem 13:21-28

30. Loffing J, Zecevic M, Feraille E et al (2001) Aldosterone induces rapid apical translocation of $\mathrm{ENaC}$ in early portion of renal collecting system: possible role of SGK. Am J Physiol Renal Physiol 280:F675-F682

31. August JT, Nelson DH, Tthorn GW (1958) Response of normal subjects to large amounts of aldosterone. J Clin Invest 37:15491555

32. Young DB, Guyton AC (1977) Steady state aldosterone doseresponse relationships. Circ Res 40:138-142

33. Frindt G, Palmer LG (2009) $\mathrm{K}^{+}$secretion in the rat kidney: $\mathrm{Na}^{+}$ channel-dependent and -independent mechanisms. Am J Physiol Renal Physiol 297:F389-F396

34. DeFronzo RA, Felig P, Ferrannini E, Wahren J (1980) Effect of graded doses of insulin on splanchnic and peripheral potassium metabolism in man. Am J Physiol 238:E421-E427

35. Diabetes Control and Complications Trial Research Group (1993) The effect of intensive treatment on the development and progression of long-term complications in insulin-dependent diabetes mellitus. N Engl J Med 329:977-986

36. Diabetes Control and Complications Trial/Epidemiology of Diabetes Interventions and Complications Research Group (2000) Retinopathy and nephropathy in patients with type 1 diabetes. N Engl J Med 342:381-389 
37. UK Prospective Diabetes Study (UKPDS) Group (1998) Intensive blood-glucose control with sulphonureas or insulin compared with conventional treatment and risk of complications in patients with type 2 diabetes (UKPDS 33). Lancet 352:837-853

38. The Diabetes Control and Complications Trial/Epidemiology of Diabetes Interventions and Complications (DCCT/EDIC) Research Group Study Research Group (2005) Intensive diabetes treatment and cardiovascular disease in patients with type 1 diabetes. N Engl J Med 353:2643-2653

39. Van den Berghe G, Wouters P, Weekers F et al (2001) Intensive insulin therapy in the critically ill patients. N Engl J Med 345:1359-1367

40. Van den Berghe G, Wilmer A, Hermans G et al (2006) Intensive insulin therapy in the medical ICU. N Engl J Med 354:449-461

41. The NICE-SUGAR Study Investigators (2009) Intensive versus conventional glucose control in critically ill patients. N Engl J Med 360:1283-1297
42. Caduff A, Talary MS, Mueller M et al (2009) Non-invasive glucose monitoring in patients with type 1 diabetes: a multisensor system combining sensors for dielectric and optical characterisation of skin. Biosens Bioelectron 24:2778-2784

43. Helfant RH (1986) Hypokalemia and arrhythmias. Am J Med $80: 13-22$

44. Gastaldelli A, Emdin M, Conforti F, Camastra S, Ferrannini E (2000) Insulin prolongs the QTc interval in humans. J Physiol Regul Integr Comp Physiol 279:R2022-R2025

45. Robinson RT, Harris ND, Ireland RH, Lee S, Newman C, Heller SR (2003) Mechanisms of abnormal cardiac repolarization during insulin-induced hypoglycemia. Diabetes 52:1469-1474

46. Tattersall RB, Gill GV (1991) Unexplained deaths of type 1 diabetic patients. Diabet Med 8:49-58

47. Thordarson H, Sovik O (1995) Dead in bed syndrome in young diabetic patients in Norway. Diabet Med 12:782-787 